\title{
Commonality in Tinnitus Perception
}

\section{Kamala S*, Jaya V and Shalini M}

Faculty of BASLP course, Madras Medical College, India

*Corresponding author: Kamala Sarathy, Faculty of BASLP course, Institute of Speech and Hearing, Madras Medical College, Chennai - 03, India, E-mail: kamalasarathy.audio@gmail.com

\section{Research Article}

Volume 2 Issue 2

Received Date: October 24, 2017

Published Date: December 11, 2017

DOI: $10.23880 /$ ooaj-16000160

\section{Abstract}

Background: Tinnitus is defined as the perception of the sound in the absence of external stimulation. Since tinnitus is a subjective phenomenon, its characteristics vary from individual to individual. A vast heterogeneity prevails in the pathophysiology of tinnitus. The phenomenon of maladaptive plasticity changes in the auditory system and Non-auditory system is widely accepted mechanism of tinnitus.

Objective: A very few studies have been to identify the stimulus most matched by the individuals with tinnitus. None of the study could explain the reason behind why a particular stimulus is perceived most by the tinnitus group amidst the heterogeneity in the subjective perception. This study is mainly done to identify the stimulus reported by tinnitus group as most mimicking their tinnitus and to explain the neurophysiology behind this homogeneity in the perception. To sort out the stimulus that is most matched by the individuals with tinnitus in tinnitus matching testing using Pure Tone Audiometer and to explore the neurophysiological mechanisms responsible for the commonality in perception of tinnitus. Method: This is the retrospective review of data obtained from Pure-tone audiometry and tinnitus evaluation results of129 individuals with different degrees of sensory-neural hearing loss. The data was analysed and expressed in terms of numbers and percentage.

Results: Among the 129 individuals, 67\% ( 83 individuals) reported Pure Tone tinnitus; 18\% ( 23 individuals) reported Narrow Band Noise tinnitus; 10\% (12 individuals) reported White Noise tinnitus; $3 \%$ (4 individuals) reported warble tone like tinnitus and remaining $2 \%$ (2 individuals) reported pulsed tone like tinnitus.

Conclusion: Through this study, we conclude the type of stimulus which is predominantly perceived in individuals with tinnitus. The hyperactivity in classical pathway is more prevalent than non- classical pathway.

Keywords: Tinnitus; Tinnitogram; Classical pathways; Non-classical pathways

Abbreviations: TMS: Transcranial Magnetic Stimulation; PTA: Pure Tone Audiometry

\section{Introduction}

The word tinnitus is derived from the Latin word 'tinnire' which means to ring like a bell. It is defined as the 


\section{Otolaryngology Open Access Journal}

phantom auditory sensation i.e., the perception of sound without corresponding acoustic or mechanical correlates in the cochlea [1]. Tinnitus represents one of the most common and distressing otologic problems which cause various somatic and psychological disorders that interfere with the quality of life [2]. Tinnitus is one of the most prevalent medical conditions in the world. It is estimated that $20-35 \%$ of the population are affected by tinnitus. A population based study of hearing loss in adults aged 48 to 92 years found that tinnitus had a prevalence of $8.2 \%$ at baseline and an incidence $5.7 \%$ during a 5 year follow up [3]. Tinnitus is categorized into subjective tinnitus and objective tinnitus. Objective tinnitus refers to sound generated by the body that are heard by the patient and also by the physician whereas subjective tinnitus is perceived by the individual alone without any external stimulus. The exact mechanism behind the perception of subjective tinnitus is poorly understood. This poor understanding of the tinnitus mechanism is mainly due to the subjective nature of the tinnitus which can be measured, quantified and described only by the responses of the individuals. However, based on the neurobiological research the most forms of tinnitus are attributable to the maladaptive plasticity changes in auditory system [4-8] and non- auditory system [9]. The maladaptive plasticity changes includes the enhanced central gain due to the compensatory increase in the central auditory activity in response to the loss of sensory input and the abnormal emotional reactions associated with the tinnitus $[10,1]$.

Very few studies have been done to find out the most common type of stimulus matched by the individual with tinnitus. None of the study could explain the reason why a particular stimulus type is perceived most. The stimulus matched with tinnitus is very important in tinnitus treatment as the amount of tinnitus suppression depends on the tinnitus characteristics and the stimulation design used. The tonal tinnitus can be suppressed equipotentially by tonal or noise stimulation in masking and tonic or burst stimulation in Transcranial Magnetic Stimulation (TMS) whereas the noise tinnitus can be suppressed only by noise stimulation in masking or by burst stimulation in Transcranial Magnetic Stimulation (TMS) [11]. The aim of the study is to identify the stimulus most perceived by the individuals with tinnitus and to establish the reason from the literature for higher prevalence of a particular stimulus over other stimulus matched by the tinnitus group.

\section{Materials and Methods}

The study included 129 patients (ears) who were suffering from unilateral continuous subjective tinnitus. The age range of the patients included in study was 11-80 years. All the patients underwent audiological Pure Tone Audiometry (PTA) and were diagnosed to have sensoryneural hearing loss and the severity ranging from mild to severe (according to World Health Organization grades of hearing impairment-WHO, 2008). The audiometric testing was carried using INVENTIS PIANO audiometer, supra aural headphones TDH-39 and the testing was carried in the acoustic treated room. The Pure Tone Audiometry testing was carried on all individuals to obtain the hearing thresholds of the individuals at each frequency i.e $125 \mathrm{~Hz}, 500 \mathrm{~Hz}, 1 \mathrm{kHz}, 2 \mathrm{kHz}, 4 \mathrm{kHz}$ and $8 \mathrm{kHz}$ in Air conduction testing and Bone Conduction testing (excluding $8 \mathrm{kHz}$ ). If threshold in any of the frequency was found to be above $25 \mathrm{~dB} \mathrm{HL}$, then it was diagnosed as hearing loss. All the patients underwent tinnitus evaluation that included pitch matching, loudness matching and octave confusion test. The patients were counseled clearly regarding the test procedure. Pure tones and Narrow Band Noises were used according to the range of loudness and frequency. 12 kinds of frequencies were used for pitch matching. The patients were instructed to match the pitch of the external tone to the pitch of the tinnitus. The tone was presented in the ear contralateral to the tinnitus ear to avoid residual inhibition. Three trials of pitch matching with an interval of 1 minute were given. Once the pitch of tinnitus was established, the patients underwent octave confusion test where they were asked to perform the pitch matching with two confusing tones. One tone was one octave above the pitch of the tinnitus established in pitch matching and other tone was one octave below the pitch of the tinnitus. The pitch matched by the individuals on all three trails with negative octave confusion test was considered as the pitch of tinnitus.

\section{Results}

Among the 129 individuals, 67\% ( 83 ears) reported Pure Tone tinnitus; $18 \%$ ( 23 ears) reported Narrow Band Noise tinnitus; $10 \%$ (12 ears) reported White Noise tinnitus; $3 \%$ (4 ears) reported warble tone like tinnitus and remaining 2\% (2 ears) reported pulsed tone like tinnitus (Figure 1). 


\section{Otolaryngology Open Access Journal}

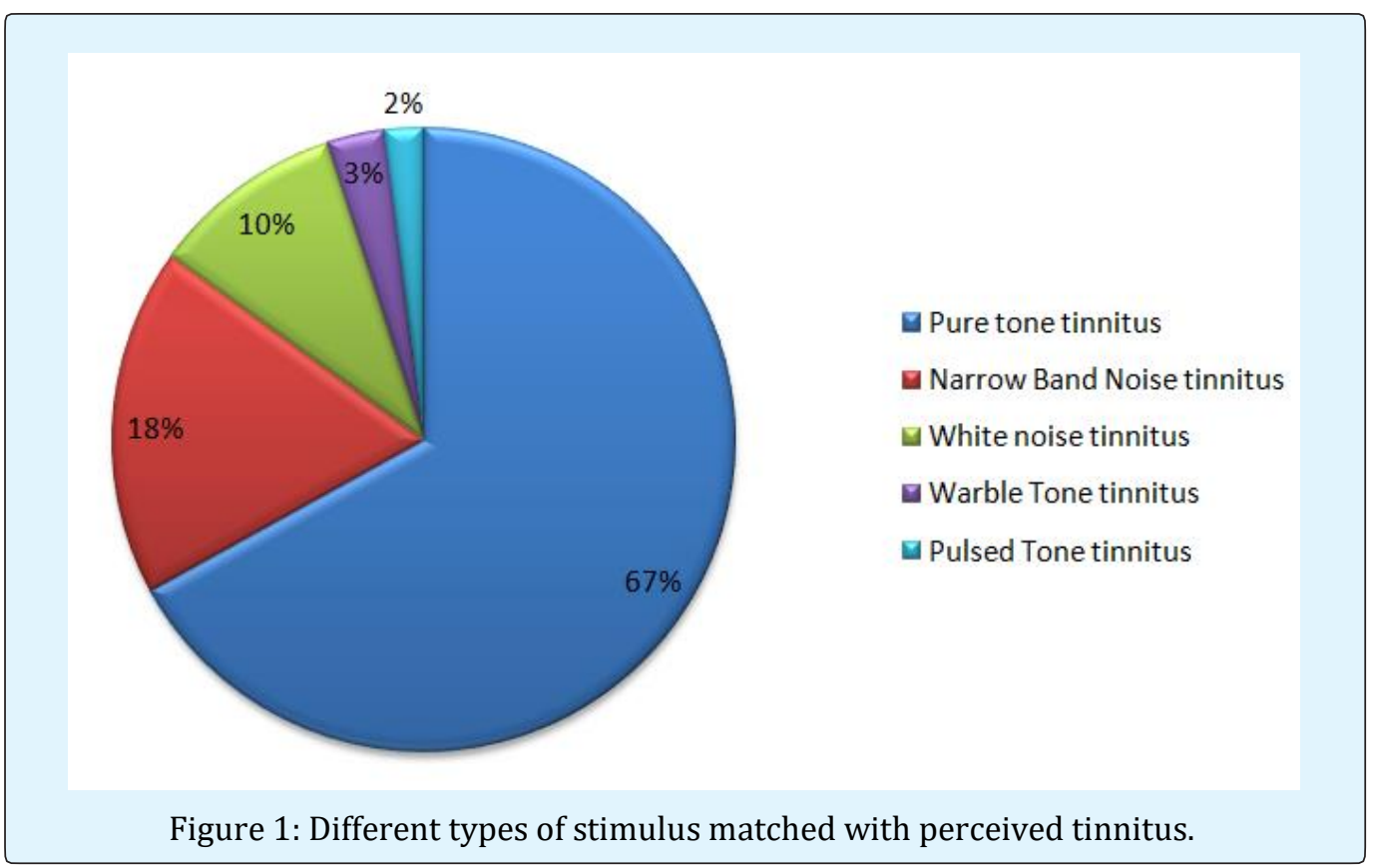

\section{Discussion}

The higher prevalence for pure tone is comparable to other study. According to the study by Eun Woong Ryu, et al. [12] in 2011 conducted on 50 individuals revealed $72 \%$ of individuals indicated pure tone tinnitus and $24 \%$ indicated narrow band noise tinnitus [12]. Another study by Larry ER, et al. [13] in 2008 conducted on 90 individuals showed $53.3 \%$ indicated tonal tinnitus and $46.6 \%$ indicated noise tinnitus [13]. Another study by Turner [14] on 1990 showed 59\% of individuals indicated tonal tinnitus and $25 \%$ indicated noise type tinnitus [14]. All these studies indicate pure tone tinnitus to be most matched by the individuals with tinnitus. However none of the studies could explain the reason for the perception of tonal tinnitus to be the most common among the tinnitus group. The subjective nature of the tinnitus and the subjective assessment of the tinnitus are the potential challenges to delineate the reason for this perception of tonal tinnitus. However few neurobiological studies have tried to reveal the neurophysiology behind the perception of tonal and noise tinnitus. A study by Sven Vanneste, et al. [15] provided Neuro-physiological differences between pure tone tinnitus and narrow band noise tinnitus using EEG findings. This study demonstrated that Narrow Band Noise tinnitus differ from the pure tone tinnitus in lateral fronto polar and Para-hippocampal areas for delta, beta and gamma frequency [15]. Another study by De Rider et al in 2007 supported the hypothesis that noise tinnitus may be caused by the increased burst firing in the nontonotopic extralemniscal system whereas pure tone tinnitus may be caused due to the increased tonic firing in the tonotopic lemniscal system through Transcranial Magnetic Stimulation (TMS) findings of 28 individuals with tinnitus (14 individuals with pure tone tinnitus and 14 individuals with narrow band noise tinnitus) [11]. This study yielded the finding that noise tinnitus is suppressed by burst stimuli and tonal tinnitus is suppressed by either tonal stimuli or burst stimuli. The classical pathway use neurons of ventral thalamus that project to the primary auditory cortex. They respond to the sensory stimulation of one modality. The non- classical pathway use medial and dorsal thalamic nuclei that project to secondary auditory cortex bypassing the primary auditory cortex. They respond to the sensory stimulation of more than one modality [11]. Neurons in ventral thalamus fire in tonic or semi tonic mode while neurons in medial or dorsal thalamus fire in bursts [16,17]. A study by Moller AR, et al. [18] have described that some forms of tinnitus may involve the extralemniscal system [18]. Through all these studies, we suggest that hyperactivity in the tonotopically organized lemniscal system is more common than that of the extralemniscal system which could be the probable reason for the perception of Pure Tone tinnitus to be more common among the individuals with subjective tinnitus.

\section{Conclusion}

Tinnitus is becoming a prevalent medical condition. The pathophysiology of tinnitus is not well explained due to the subjective nature and heterogeneity in the 


\section{Otolaryngology Open Access Journal}

characteristics of tinnitus. The present study was aimed to identify the homogeneity in the stimulus perception in tinnitus group. Through this study, we conclude that Pure Tone tinnitus is most common followed by the Narrow Band Noise tinnitus and White Noise tinnitus. We also conclude that the pure tone tinnitus is may be due to the hyperactivity in Lemniscal system while the Noise tinnitus may be due to the hyperactivity in ExtraLemniscal System.

\section{References}

1. Jastreboff PJ (1990) Phantom Auditory Perception (Tinnitus): Mechanisms of Generation and Perception. Neurosci Res 8(4): 221-254.

2. Yetsier S, Tosun F, Satar B, Arslanhan M, Akcam T, et al. (2002) The Role of Zinc in Management of tinnitus. Auris Nasus Larynx 29(4): 329-333.

3. Nondahl DM, Cruickshanks KJ, Wiley TL, Klein R, Klein BE, et al. (2002) Prevalence and 5-Year Incidence of Tinnitus among Older Adults: The Epidemiology of Hearing Loss Study. J Am Acad Audiol 13(6): 323-331.

4. Weisz N, Dohrmann K, Elbert T (2007) The Relevance of Spontaneous Activity for the Coding of the Tinnitus Sensation. Prog Brain Res 166: 61-70.

5. Cacace AT, Cousins JP, Parnes SM, McFarland DJ, Semenoff D, et al. (1999) Cutaneous- evoked Tinnitus II- Review of Neuroanatomical, Physiological and Functional Imaging Studies. Audiol Neurootol 4(5): 258-268.

6. Mirz F, Pedersen B, Ishizu K, Johannsen P, Ovesen T, et al. (1999) Positron Emission Tomography of Centers of Tinnitus. Hear Res 134(1-2): 133-144.

7. Lockwood AH, Salvi RJ, Coad ML, Towsley ML, Wack DS, et al. (1998) The Functional Neuroanatomy of Tinnitus: Evidence For Limbic System Links And Neural Plasticity. Neurology 50(1): 114-120.

8. Smits M, Kovacs S, de Ridder D, Peeters RR, van Hecke $P$, et al. (2007) Lateralization of Functional Magnetic Resonance Imaging (fMRI) Activation in the Auditory Pathway of Patients with Lateralized Tinnitus. Neuroradiology 49(8): 669-679.
9. Andersson G, Lyttkens L, Hirvela C, Furmark T, Tillfors M, et al. (2000) Regional Cerebral Blood Flow During Tinnitus: A PET Case Study With Lidocaine and Auditory Stimulation. Acta Otolaryngol 120(8): 967-972.

10. Benjamin D Auerbach, Paulo V Rodrigues, Richard J Salvi (2014) Central Gain Control in Tinnitus and Hyperacusis. Front Neurol 5: 206.

11. De ridder D, van der Loo E, van der Kelen K, Menovsky T, van de Heyning P, et al. (2007) Do Tonic Or Burst TMS Modulate The Lemniscal And Extralemniscal System Differentially?. Int J Med Sci 4(5): 242-246.

12. Eun Woong Ryu, Chul Kwon, Cheol Eon Park, Jae Yong Byun, Seung Geun Yeo, et al. (2011) Possibility of Discriminating the Presence of Tinnitus through the Repeated Tinnitogram. Korean J Audiol 15(3): 119123.

13. Larry E Roberts, Graeme Moffat, Michael Baumann, Lawrence M Ward, Daniel J Bosnyak (2008) Residual Inhibition Functions Overlap Tinnitus Spectra And The Region Of Auditory Threshold Shift. J Assoc Res Otolaryngol 9(4): 417-435.

14. John Turner SJR (1990) Auditory Dysfunction: Tinnitus, Clinical Methods: The History, Physical, and Laboratory Examination. $3^{\text {rd }}$ (edn), Boston: Butterworths Chapter 121.

15. Sven Venneste, Mark Plazier, Elsa van der Loo, Paul Van de Heyning, Dirk De Ridder, The Differences in Brain Activity between Narrow Band Noise Tinnitus and Pure Tone Tinnitus. PLos One 5(10): 13618.

16. He J, Hu B (2002) Differential Distribution of burst and single-spike responses in auditory thalamus. J Neurophysiol 88(4): 2152-2156.

17. Hu B, Sentorov V, Mooney D (1994) Lemniscal and Non- Lemniscal Synaptic Transmission in Rat Auditory Thalamus. J Physiol 479(2): 217-231.

18. Moller AR, Moller MB, Yokota M (1992) Some Forms of Tinnitus may involve the Extralemniscal Auditory Pathway. Laryngoscope 102(10): 1165-1171. 